\title{
Simulation of a New Endcap Time of Flight System for the BESIII Experiment
}

\author{
M. Ullrich*†, S. Braun, J. Hu, W. Kühn, S. Lange, Y. Liang, B. Spruck, M. Werner and \\ H. Ye \\ for the BESIII Collaboration
}

Justus-Liebig-Universität Giessen

II. Physikalisches Institut

Heinrich-Buff-Ring 16

D-35392 Giessen

\begin{abstract}
We present the results of a full simulation of a Multigap-Resistive Plate-Chamber (MRPC) with an intrinsic time resolution of about $45 \mathrm{ps}$ for the BESIII (Beijing Spectrometer III) detector [1]. This type of time-of-flight (TOF) detector is discussed to replace the actual endcap TOF detector, which provides a total time-resolution of 148 ps for electrons in Bhabha scattering events.

The MRPC has been implemented into the BESIII offline software system (BOSS) [2] ; which is built on the Gaudi-architecture using the object-orientated language $\mathrm{C}++$. The detailed simulation code is based on Geant 4 [3, 4], the implemented reconstruction code allows a full matching with all other sub-detectors of the BESIII experiment.

It is shown that with such type of detector a total time resolution of about $80 \mathrm{ps}$ can be achieved, allowing a pion/kaon separation up to a momentum of $1.5 \mathrm{GeV} / \mathrm{c}$ at a $2 \sigma$ level ( $\hat{=} 7.9 \%$ misidentification).
\end{abstract}

LI International Winter Meeting on Nuclear Physics

21-25 January 2013

BORMIO, Italy

\footnotetext{
*Speaker.

†E-mail: Matthias.Ullrich@physik.uni-giessen.de
} 


\section{Introduction}

The BESIII experiment is located at the Beijing Electron-Positron Collider II (BEPC II) in Beijing, China at the Institute for High Energy Physics, commonly known as IHEP. The symmetric $\mathrm{e}^{+} \mathrm{e}^{-}$experiment optimized for the investigation of $\tau$ and charm physics $(1.8 \mathrm{GeV} \leq \sqrt{s} \leq$ $4.2 \mathrm{GeV}$ ) is designed for a peak luminosity of $10^{33} \mathrm{~cm}^{-2} \mathrm{~s}^{-1}$ and has an acceptance of $93 \%$ of $4 \pi$. The innermost detector at the BESIII detector is a helium gas based multi-wire drift chamber (MDC) providing a spatial resolution of $\approx 135 \mu \mathrm{m}$, a momentum resolution better than $0.5 \%$ for charged tracks and a dE/dx resolution better than $6 \%$. The next outer detector is the TOF system made of plastic-scintillator with a time resolution in the barrel part of about $80 \mathrm{ps}$ and about $150 \mathrm{ps}$ in the endcap region. The TOF system is surrounded by a CsI(Tl) electro-magnetic calorimeter (EMC) for electron identification and photon detection with an energy resolution of $2.5 \%$ at $1 \mathrm{GeV}$ in the barrel part and $6 \%$ in the endcap part.

The 3 inner detectors are encased by a superconducting magnet providing a magnetic field of $1.0 \mathrm{~T}$ and a resistive plate counter muon detector modules (MUC) with a spatial resolution of $\approx 2 \mathrm{~cm}$ $[1,5]$.

It is discussed to replace the endcap TOF detectors by multi-gap resistive plate chambers (MRPC). The system will consist of 4 layers ( 2 for each endcap) each containing 18 modules with 24 readouts strips (compare to figure 1(a)). Those detectors will be operated at an electric field strength of about $110 \mathrm{kV}$ with as gas mixture of $90 \% \mathrm{C}_{2} \mathrm{~F}_{4} \mathrm{H}_{2}, 5 \% \mathrm{SF}_{6}$ and $5 \%$ iso-butane $\left(\mathrm{C}_{4} \mathrm{H}_{10}\right)$ and will provide a total time-resolution of less than $80 \mathrm{ps}$ for charged particles.

The differential signals generated by the MRPC are planed to be read out by boards based on NINO chips [6]. These chips amplify the signals and encode the input charge into the width of the produced LVDS signals. Those signals can be read out by TDC boards based on the HPTDC chip, which can measure both the time of the leading and the trailing edge with a resolution of about 27 ps $[7,8]$. The pulse length (time over threshold) can be used for an offline slewing correction later on.

\section{Multigap-Resistive-Plate Chambers}

MRPCs are gas based avalanche detectors composed of a stack of resistive plates with a high bulk resistivity (between $10^{11}$ and $10^{13} \Omega \mathrm{cm}$ ) which are separated by the mean of spacers creating a series of equally sized gas gaps. Electrodes are connected to the outer surface while the inner plates are kept electrically floating.

Such a detector system is well suited for time of flight systems as it provides an excellent intrinsic time resolution $(\ll 100 \mathrm{ps})$, a high detection efficiency $(>99 \%)$ and a high rate capability $(\approx 500$ $\mathrm{Hz} / \mathrm{cm}^{2}$ ) [9]. A schematic view of a double-stack MRPC is given in figure 1(a).

\section{Simulation}

The detectors geometry and element properties as well as the one of the supporting aluminum boxes (compare to figure 1(b)) which contain the front-end-electronics (FEE) and the detector itself 


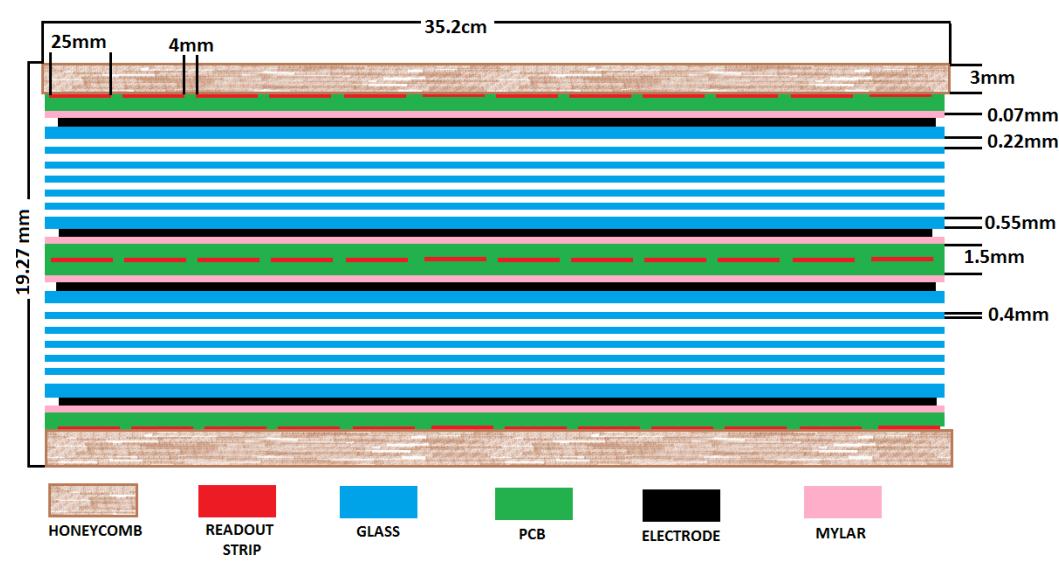

(a)

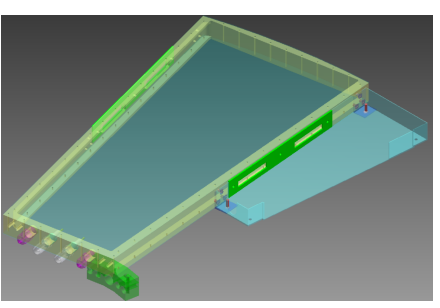

(b)

Figure 1: (a) A schematic side view of the MRPC which may be installed in the BESIII experiment. (b) Supporting structure of the planed MRPC upgrade: The larger box will contain the MRPC itself, the smaller one the front-end-electronics.

are implemented into BOSS using the Geant 4 concept of logical and physical volumes.

The charged signal production based on an avalanche gas model developed by W. Legler [10] allows a fast and realistic avalanche simulation. The method is outlined here briefly: Electron multiplication in high electric fields can be described using statistical language. Starting from one electron at the spatial coordinate $x=0$ the probability to have $n$ electrons at $x+d x$ is:

$$
P(n)=\left\{\begin{array}{lr}
k \bar{n} \bar{n}(d x)-1 & \text { for } n=0 \\
\bar{n}(d x)-k & \left.\frac{1-k}{\bar{n}(d x)-k}\right)^{2}(k \bar{n}(d x)-1 \\
\bar{n}(d x)-k & \text { for } n>0
\end{array}\right.
$$

with $\bar{n}(d x)=\exp ((\alpha-\eta) d x), k=\eta / \alpha, \alpha$ the Townsend-coefficient and $\eta$ the attachment-coefficient. The MRPC works under a high electric field, i.e. the solution for $\alpha=\beta$ and $\alpha=0$ can be neglected. The variance of this distribution is $\sigma(x)^{2}=\frac{1+k}{1-k} \bar{n}(x)(\bar{n}(x)-1)$. To generate random numbers according to this equation, one draws a random number $s$ out of $(0,1)$ and calculates

$$
\begin{array}{rlr}
n & =0 ; & s<\frac{\bar{n}(d x)-1}{\bar{n}(d x)-k} \\
n=1+\operatorname{int}\left[\frac{1}{\ln \left(\frac{\bar{n}(d x)-1}{\bar{n}(d x)-k}\right)} \ln \left(\frac{(\bar{n}(d x)-k)(s-1)}{(k-1) \bar{n}(d x)}\right)\right] ; & \text { else . }
\end{array}
$$

int means here the truncation of decimals.

As the evaluation of the logarithm is very time consuming, for sufficient large number of $n(=150)$ a different technique is applied: The electrons at some distance $x+d x$ can be obtained by drawing a random number from a Gaussian distribution with mean $\mu=n(x) \bar{n}(d x)$ and standard deviation $\sigma=\sqrt{n(x)} \sigma(d x)$.

In order to take into account the space charge effects, a saturation level for an avalanche is defined to be of $1.5 \cdot 10^{7}$ electrons [11] [12].

This approximate method of simulation is sufficient, as the main characteristics such as efficiency 
and time resolution are basically not affected by the space charge - they are only sensitive to the early stage of the avalanche where the effect can be neglected [13].

The induced current into the readout-strips is calculated utilizing Ramo's theorem of induction: $I(t)=E_{\text {weight }} v_{d r i f t} Q_{e} N(t)$ with $N(t)$ the number of electrons at time $t$ and $v_{d r i f t}$ the drift velocity of the electrons. The weighting field $E_{\text {weight }}$ can be expressed by

$E_{\text {weight }}=1 /\left\{n_{\text {gap }} d_{\text {gap }}+\left(\left(n_{\text {gap }}-1\right) d_{\text {in }}+2 d_{\text {out }}\right) / \varepsilon_{\text {Glass }}+2 d_{\text {Mylar }} / \varepsilon_{\text {Mylar }}\right\} \quad$ [12] (compare to figure 1(a)). $n_{\text {gap }}$ is the number of gaps, $d_{\text {gap }}$ the width of the gap, $d_{i n}, d_{\text {out }}$ and $d_{\text {Mylar }}$ the thickness of inner, outer resistive plate and Mylar tape, while $\varepsilon$ is the relative permittivity of the materials. The Townsend-coefficient $\alpha$ and the attachment-coefficient $\eta$ have been calculated with MAGBOLTZ [14] for a gas mixture of $90 \% \mathrm{C}_{2} \mathrm{~F}_{4} \mathrm{H}_{2}, 5 \% \mathrm{SF}_{6}$ and $5 \%$ iso-butane. The results are shown in figure 4.

Uncertainties arising from the electronic components (FEE, time-to-digital conversion (TDC), cables, ...) are considered by using their experimentally determined values (smeared with a gaussian distribution).

The results of the model have been compared with the data measured during a beam test with a MRPC prototype at the BEPC E3 beam line; the incident particles have been mainly protons with a momentum of about $600 \mathrm{MeV} / \mathrm{c}$ [15]. The comparison of measured and simulated data are shown in figure 2 and 3.

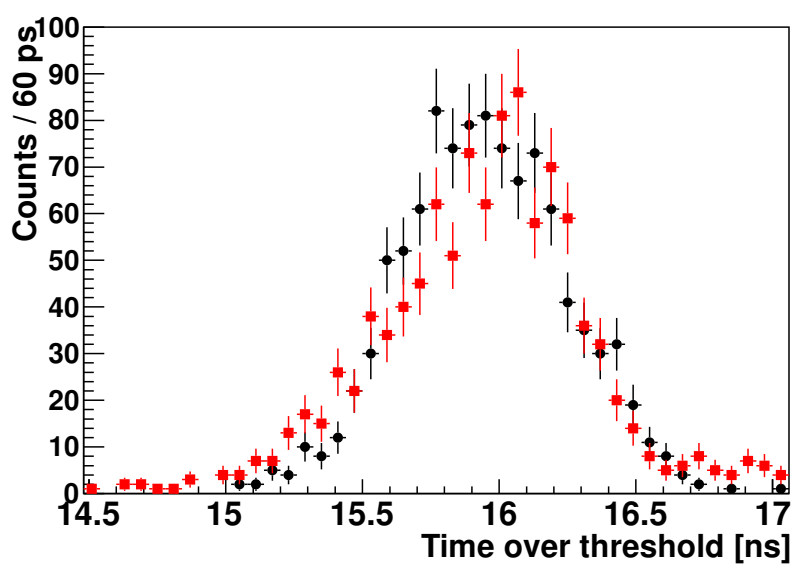

Figure 2: Time over threshold (measured data in red, simulation in black), electrical field $\mathrm{E}=112 \mathrm{kV} / \mathrm{cm}$

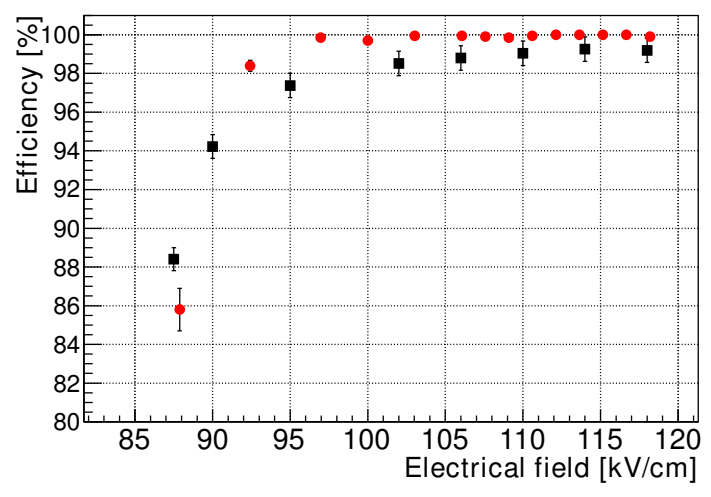

(a)

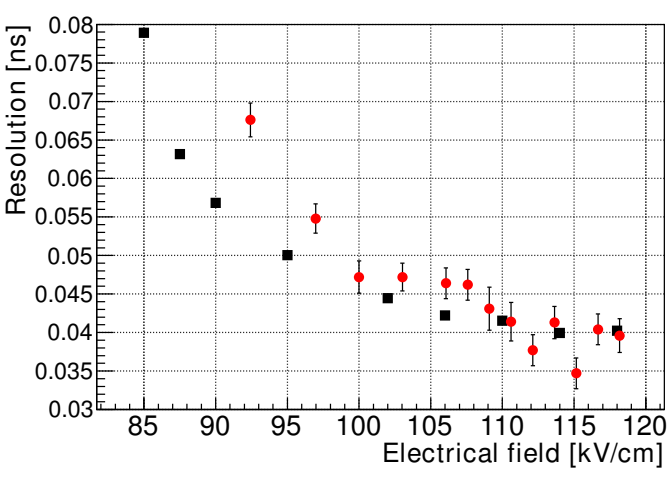

(b)

Figure 3: Comparsion of measured data (red) and simulated (black) data for protons with a momentum of about $600 \mathrm{MeV} / \mathrm{c}$ [15].

The measured data matches the simulated one for electrical field strength larger than 100 $\mathrm{kV} / \mathrm{cm}$, for field strength smaller than this value the time resolution is underestimated and the efficiency is overestimated (compare to figure 3). In this area recombination and space charge 
effects, which are considered only as an approximation in this simple model, play an important role. However, the MRPCs are planned to be operated at electrical field strength larger than 110 $\mathrm{kV} / \mathrm{cm}$.

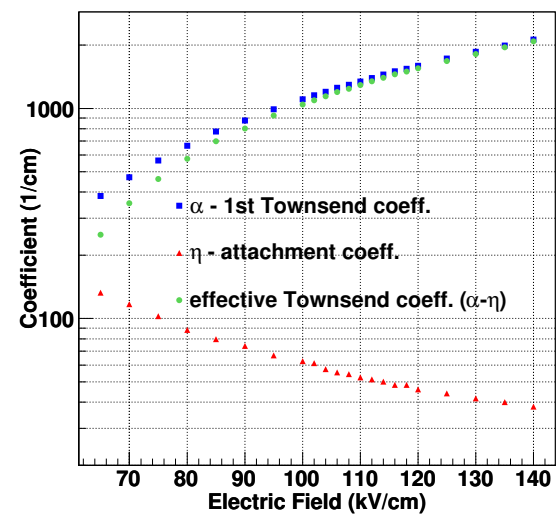

(a)

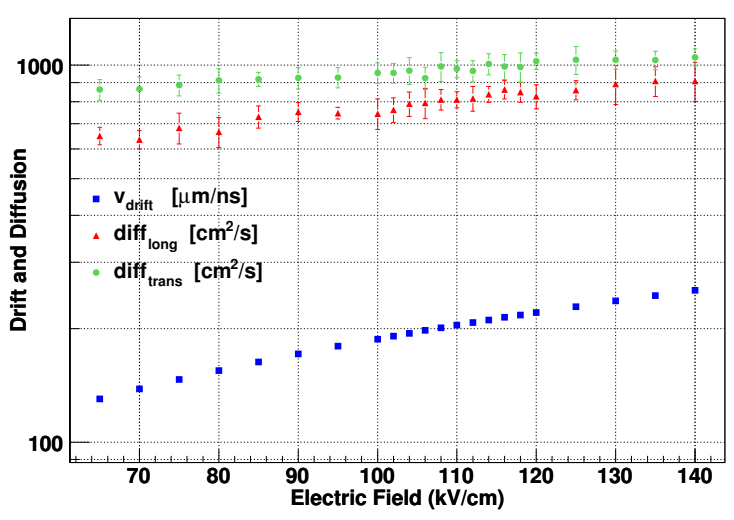

(b)

Figure 4: Townsend-coefficient $\alpha$, attachment-coefficient $\eta$, electron drift velocity and longitudinal and transverse diffusion coefficients for a gas mixture of $90 \% \mathrm{C}_{2} \mathrm{~F}_{4} \mathrm{H}_{2}, 5 \% \mathrm{SF}_{6}$ and $5 \%$ iso-butane as a function of the electric field calculated with MAGBOLTZ.

\section{Reconstruction}

In order to use the measured time information in the BESIII detector for particle identification, the tracks of the MDC have to be matched with the MRPC's signals.

A Geant 4 based algorithm, which considers magnetic deflections and ionization-loss of charged particles, extrapolates the MDC tracks to the outer sub-detectors. In the neighborhood of the extrapolated hit position on the MRPC cell it is searched for signals of the MRPC detector. The size of the region to be searched in can be defined by the user. If more than two possible MRPC signals are found to match one MDC track, the selected one is the one with the largest deposition of charge, i.e. by the largest time-over-threshold.

The extrapolated position is also used to correct for the signal transition time within one readout strip, as the 24 readout strips of the implemented version of the MRPC modules are only read out at one side (compare to figure 5).

This method of correction can only be applied, if the extrapolated cell is the one which has caused an signal. For all other cases a correction based one the geometrical size of the readout strip is applied.

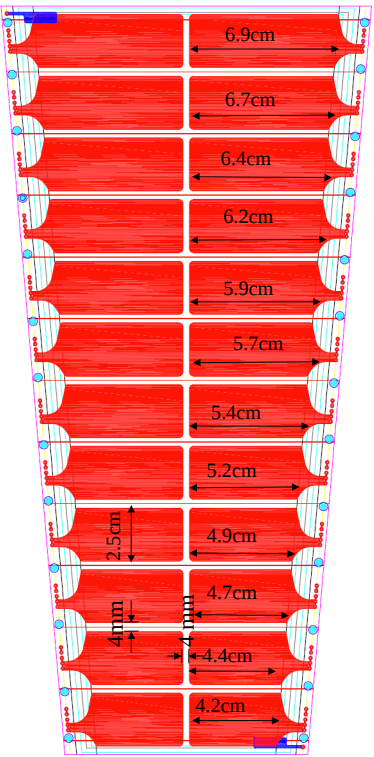

Figure 5: Sketch of the readout-pads and their dimension 
A comparison of the simulated and the reconstructed transition time reveals a resolution of about 22 ps (corresponding to a position resolution for the extrapolation of $0.5 \mathrm{~cm}$ ) for signals, where the extrapolated track was usable.

\section{Results}

One important result is the improvement in pion/kaon discrimination which one can expect due to the gain in time resolution. The interesting distribution to determine is difference in measured and expected time $\Delta T=t_{\text {meas. }}-t_{\text {exp }}$, where $t_{\text {meas. }}$ is the measured time of flight and $t_{\text {exp. }}$ the expected time of flight. The expected time of flight for a particle can be calculated by

$$
t_{\text {exp. }}=\frac{L}{\beta \cdot c}=\frac{L}{\frac{p / m}{\sqrt{1+p^{2} / m^{2}}} \cdot c},
$$

where $L$ is the extrapolated track length, $p$ the momentum information determined by the MDC, $m$ the particle's mass, $\beta$ the relative velocity and $c$ the velocity of light.

Calculating $\Delta T$ once assuming the particle was a pion and once the particle was kaon, the correct distribution should peak around zero, whereas the wrong assumption should peak left or right from zero. Figure 6(a) shows such a distribution for pions with a momentum between $1.2 \mathrm{GeV} / \mathrm{c}$ and 1.3 $\mathrm{GeV} / \mathrm{c}$ : Assuming that the particle was an kaon yields a distribution around -0.35 ns whereas the correct assumptions shows a peak around $0 \mathrm{~ns}$.

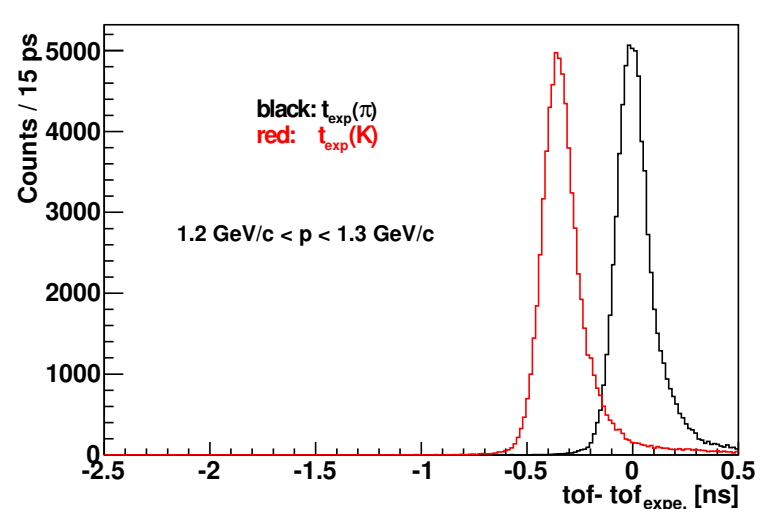

(a)

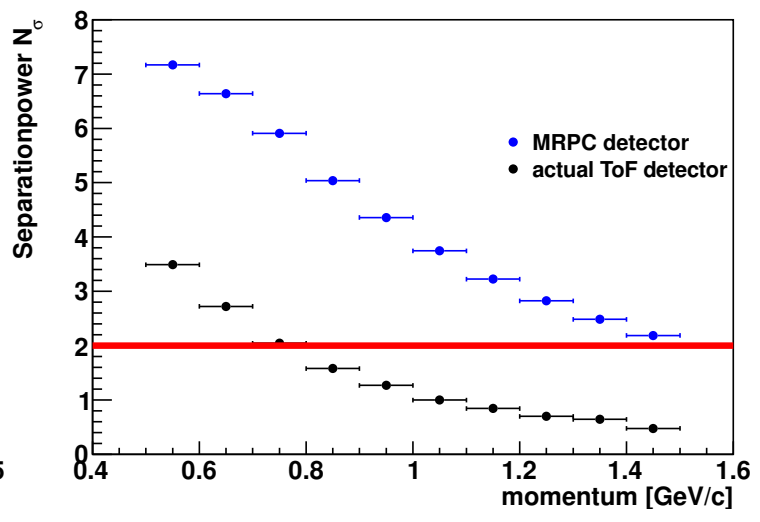

(b)

Figure 6: (a) Measured time of flight minus expected time of flight for pions with momenta between $1.2 \mathrm{GeV} / \mathrm{c}$ and $1.3 \mathrm{GeV} / \mathrm{c}$ once assuming the particle is a pion and once a kaon. (b) A comparison of the separation powers of the actual TOF detector and the planed MRPC upgrade.

Distribution similar to the one in figure 6(a) have been evaluated for pions and kaons with momenta between $0.5 \mathrm{GeV} / \mathrm{c}$ and $1.5 \mathrm{GeV} / \mathrm{c}$. They have been fitted with a gaussian kernel and the fitted values have been used to calculate the so-called separation power:

$$
N_{\sigma}=\frac{\left|\left(t_{\text {meas. }}-t_{\text {exp. }}^{\pi}\right)-\left(t_{\text {meas. }}-t_{\text {exp. }}^{K}\right)\right|}{\sqrt{\sigma_{\pi}^{2}-\sigma_{K}^{2}}},
$$


with $t_{\text {meas. }}$ the measured time of flight, $t_{\text {exp }}$. the expected time of flight for the corresponding particle and $\sigma_{K}$ and $\sigma_{\pi}$ the width of the fitted gaussian distributions. A separation power of 2 corresponds here to a misidentification of $7.9 \%$. The separation powers for the actual TOF detector and the planned MRPC upgrade are compared in figure 6(b): The MRPC detector will allow a pion/kaon discrimination up to a momentum of $1.5 \mathrm{GeV} / \mathrm{c}$ at a $2 \sigma$ level.

\section{Summary}

The simulation and reconstruction software for a new MRPC detector as possible replacement of the actual endcap TOF of the BESIII experiment has been implemented in the BESIII offline software system BOSS.

The simulation part allows a fast and realistic simulation. The simulated efficiency, time resolution and time-over-threshold agree with the data measured during beam test with a MRPC prototype at the BEPC E3 beam line.

After a full reconstruction of the data and a matching with other sub-detectors of the BESIII experiment, the improvement which is to expect in pion/kaon discrimination is determined to be at a $2 \sigma$ level up to momenta of $1.5 \mathrm{GeV} / \mathrm{c}$.

\section{References}

[1] M. Ablikim et al. (BESIII Collaboration), Nucl. Instr. and Meth. A 614(2010)345.

[2] Z. Deng, H. Liu, G. Cao, M. He, Y. Yuan, Z. You and Y. Liang, PoS ACAT (2007) 043.

[3] S. Agostinelli, J. Allison, K. Amako et al. , Nucl. Instr. and Meth. A 506(2003)250

[4] Allison, J.; Amako, K.; Apostolakis, J. et al. , Nuclear Science, Vol.53, no.1, pp.270-278, Feb. 2006

[5] arXiv:0809.1869 [hep-ex]

[6] F. Anghinolfi, P. Jarron, A.N. Martemiyanov et al., ucl. Instr. and Meth. A 533(2004)183

[7] J. Christiansen, "HPTDC Version 2.1", July 2002

[8] CAEN, Technical Information Manual, Revision n. 15, 10 July 2012 Mod. V1290-VX1290 A/N, 32/16 Ch. Multihit TDC

[9] E Cerron Zeballos et al., Nucl. Instr. and Meth. A, 374 132, 1996

[10] W. Legler, Die Statistik der Elektronenlawinen in elektronegativen Gasen, bei hohen Feldstärken und bei hoher Gasverstärkung (the statistics of electron avalanchses in electronegative gases at high electric fields an at large gas gain), Z. Naturforschung, 16a:253-261, 1961

[11] C. Lippmann and W. Riegler, Nucl. Instr. and Meth. A, 517 54-76, 2004

[12] Ming Shao et al., Meas. Sci. Technol. 17(2006) 123-127

[13] M. Abbrescia et al., CMS Conference Report CMS CR 1998/021

[14] S. Biagi, Nucl. Instr. and Meth. A, 283716,1989

S. Biagi, Nucl. Instr. and Meth. A, 421 234-240, 1999

[15] S. Yang, Y.J. Sun, C. Li et al., MRPC prototypes for the BESIII end-cap time-of-flight system upgrade, submitted to NIM A 